\title{
Radially Symmetric Steady State Thermal and Mechanical Stresses of a Poro FGM Hollow Sphere
}

\author{
M. Jabbari, ${ }^{1}$ S. Karampour, ${ }^{2}$ and M. R. Eslami ${ }^{3}$ \\ ${ }^{1}$ South Tehran Branch, Islamic Azad University, Iran \\ ${ }^{2}$ Department of Mechanical Engineering, Omidiye Branch, Islamic Azad University, Omidiye, Iran \\ ${ }^{3}$ Mechanical Department, Distinguished Thermoelastic Center, Amirkabir University of Technology, Tehran, Iran
}

Correspondence should be addressed to M. Jabbari, projectsjabbari@gmail.com

Received 7 April 2011; Accepted 5 May 2011

Academic Editor: M. Al-Nimr

Copyright (C) 2011 M. Jabbari et al. This is an open access article distributed under the Creative Commons Attribution License, which permits unrestricted use, distribution, and reproduction in any medium, provided the original work is properly cited.

\begin{abstract}
A general solution for the one-dimensional steady-state thermal and mechanical stresses in a hollow thick sphere made of porous functionally graded material (FGPM) is presented. The temperature distribution is assumed to be a function of radius, with general thermal and mechanical boundary conditions on the inside and outside surfaces of the sphere. The material properties, except Poisson's ratio, are assumed to vary along the radius $r$ according to a power law function. The analytical solution of the heat conduction equation and the Navier equation lead to the temperature profile, radial displacement, radial stress, and hoop stress as a function of radial direction.
\end{abstract}

\section{Introduction}

Functionally graded materials (FGMs) are made of a mixture with arbitrary composition of two different materials, and the volume fraction of each material changes continuously and gradually. The FGMs concept is applicable to many industrial fields such as chemical plants, electronics, and biomaterials [1]. Thick hollow sphere analysis made of FGM under mechanical and thermal loads and in asymmetric and two-dimensional $(r, \theta)$ state was conducted investigating navier equations and using legendre polynomials [2]. The analytical solution for the stresses in spheres and cylinders made of functionally graded materials are given by Lutz and Zimmerman [3, 4]. They considered thick spheres and cylinders under radial thermal loads, where radially graded materials with linear composition of the constituent materials were considered. Obata and Noda [5] studied one-dimensional steady thermal stresses in a functionally graded circular hollow cylinder and a hollow sphere using the perturbation method. The transient thermal stresses in a plate made of FGM are presented by the same authors [6]. By introducing the theory of laminated composites, Ootao and Tanigawa derived the three-dimensional transient thermal stresses of a nonhomogeneous hollow sphere with a rotating heat source [7]. Jabbari et al. presented the analytical solution of one and two-dimensional steady state thermoelastic problems of the FGM cylinder [8]. Two-dimensional nonaxisymmetric transient mechanical and thermal stresses in a thick hollow cylinder is presented by Jabbari et al. [9]. Shao and Wang presented three-dimensional solution to obtain stress fields in an FGM cylindrical plate with a finite length which is mechanical and thermal load tests and showed the results an a graph [10]. The transient thermal stress problem in a hollow sphere with homogeneous and isotropic properties is analytically solved by Cheung et al. [11]. Their assumed thermal boundary conditions are varied along the $\gamma$ direction and the problem is solved with the potential function method. Takeuti and Tanigawa have employed the potential function method to obtain the analytical solution of a homogeneous spherical vessel with rotating heat source [12]. They considered the general form of thermal boundary conditions and analyzed the problem in transient condition with the potential functions method.

Porous spheres of nanometer to micrometer dimensions are being pursued with great interest because of several possible technical applications in catalysis, drug delivery systems, separation techniques, photonics, as well as piezoelectric and other dielectric devices $[13,14]$. The study of 
the thermomechanical response of fluid saturated porous materials is important for several branches of engineering [15-19]. Some of the important cases are the disposal of high-level radioactive waste, the extraction of oil or geothermal energy, the storage of hot fluids, and road subgrade or furnace foundations, which are usually subjected to cyclic changes of temperature. Wang and Papamichos $[20,21]$ presented analytical solutions for the temperature, pore pressure and stresses around a cylindrical wellbore and a spherical cavity subjected to a constant temperature change and a constant fluid flow rate by coupling the conductive heat transfer with the pore-fluid flow. Using the so-called heat source function method. Kurashige [22] analyzed the heat and fluid flow problem of a spherical cavity subjected to a constant heat flux in an impermeable medium.

Inspite of conducted studies on spherical and cylindrical vessel made of FGM to obtain mechanical displacements and mechanical and thermal stresses, there is not any study on composition of poro and FGM materials. This study investigates the effect of mechanical and thermal stresses on one-dimensional steady state in the poro FGM hollow sphere that fluid trapped in the poro medium which is located in undrained conditions.

\section{Analysis}

Consider a thick hollow sphere of inside radius $a$ and outside radius $b$ made of poro FGM. The sphere material is graded through the radial $r$-direction. Thus, the material properties are functions of $r$. Let $u$ be the displacement component along the radial direction. The strain-displacement relations are

$$
\begin{gathered}
\varepsilon_{r r}=u_{, r}, \\
\varepsilon_{\theta \theta}=\varepsilon_{\phi \phi}=\frac{u}{r} .
\end{gathered}
$$

The stress-strain relations one-dimensional in the poro FGM hollow sphere with fluid trapped in the poro pure are

$$
\begin{gathered}
\sigma_{r r}=C_{11} \varepsilon_{r r}+2 C_{12} \varepsilon_{\theta \theta}-\gamma p \delta_{r r}-z_{r} T(r), \\
\sigma_{\theta \theta}=\sigma_{\phi \phi}=C_{12} \varepsilon_{r r}+\left(C_{22}+C_{23}\right) \varepsilon_{\theta \theta}-\gamma p \delta_{\theta \theta}-z_{\theta} T(r),
\end{gathered}
$$

where

$$
\begin{gathered}
z_{r}=C_{11} \alpha_{r}+2 C_{12} \alpha_{\theta}, \\
z_{\theta}=C_{12} \alpha_{r}+\left(C_{22}+C_{23}\right) \alpha_{\theta}, \\
\alpha_{r}=\alpha_{\theta},
\end{gathered}
$$

where $\sigma_{i j}$ and $\varepsilon_{i j}(i, j=r, \theta)$ are the stress and strain tensors, $T(r)$ is the temperature distribution determined from the heat conduction equation, $\alpha_{i}$ is the coefficient of thermal expansion of effective stress, and $C_{i j}, \gamma, p$, and $\delta_{i i}(i=r, \theta)$ are elastic constants, Biots coefficient of effective stress, poro pressure, and kronecker delta.

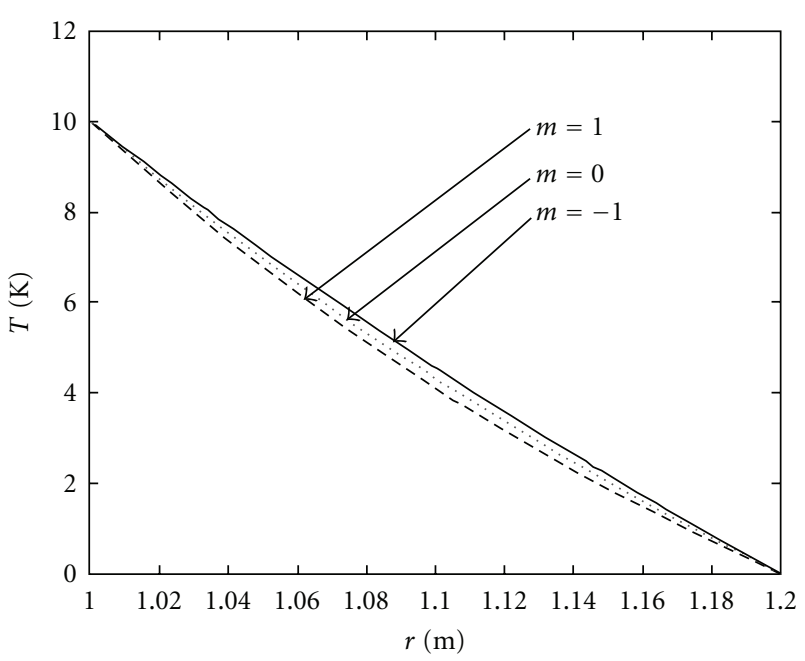

FIgURE 1: Radial temperature distribution.

Equations in a porous material are

$$
\begin{gathered}
p=M(\zeta-\gamma \in), \\
M=\frac{k_{u}-k}{\gamma^{2}}, \\
B=\frac{k_{u}-k}{\gamma k_{u}}, \\
k_{u}=k\left[1+\frac{\gamma^{2} k_{f}}{(1-\gamma)\left(\gamma-\phi_{p}\right) k_{f}+\phi_{p} k_{f}}\right] .
\end{gathered}
$$

For fluid in undrained condition,

$$
\begin{gathered}
\zeta=0, \\
p=-\gamma M \in=-\gamma M\left(\varepsilon_{r r}+\varepsilon_{\theta \theta}+\varepsilon_{\phi \phi}\right), \\
\varepsilon_{\theta \theta}=\varepsilon_{\phi \phi},
\end{gathered}
$$

where $\zeta, \in, M, k_{u}, k, k_{f}, B$, and $\phi_{p}$ are variation of fluid content, volumetric strain, Biots modulus, undrained bulk modulus, drained bulk modulus, bulk modulus of fluid, compressibility coefficient, and porosity.

The equilibrium equation in the radial direction, disregarding the body force and the inertia term, is

$$
\sigma_{r r, r}+\frac{2}{r}\left(\sigma_{r r}-\sigma_{\theta \theta}\right)=0 .
$$

Substituting (5) into (2) leads to

$$
\begin{gathered}
\sigma_{r r}=c_{1}^{*} \varepsilon_{r r}+c_{2}^{*} \varepsilon_{\theta \theta}-z_{r} T(r), \\
\sigma_{\theta \theta}=\sigma_{\phi \phi}=c_{3}^{*} \varepsilon_{r r}+c_{4}^{*} \varepsilon_{\theta \theta}-z_{\theta} T(r),
\end{gathered}
$$




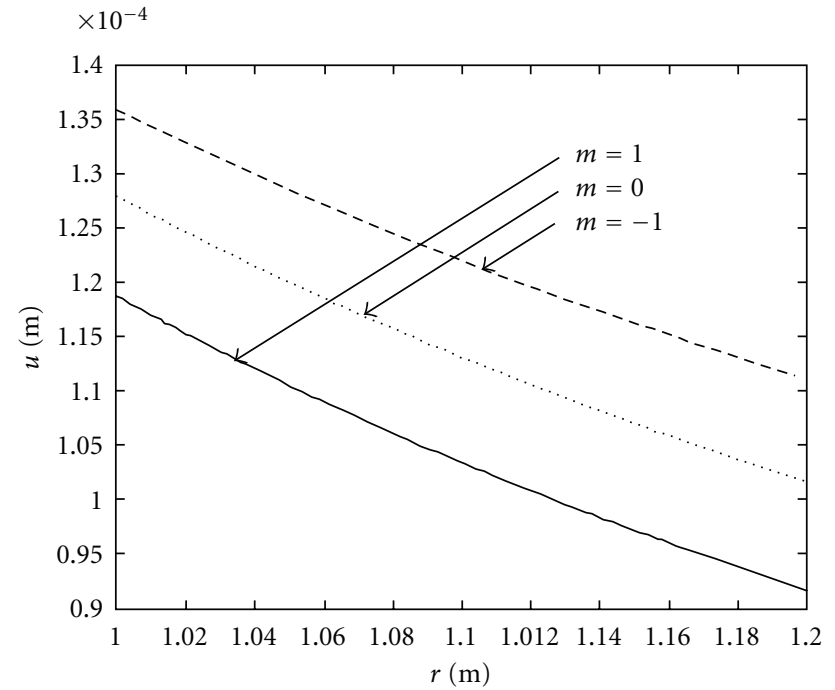

FIgure 2: Radial displacement distribution.

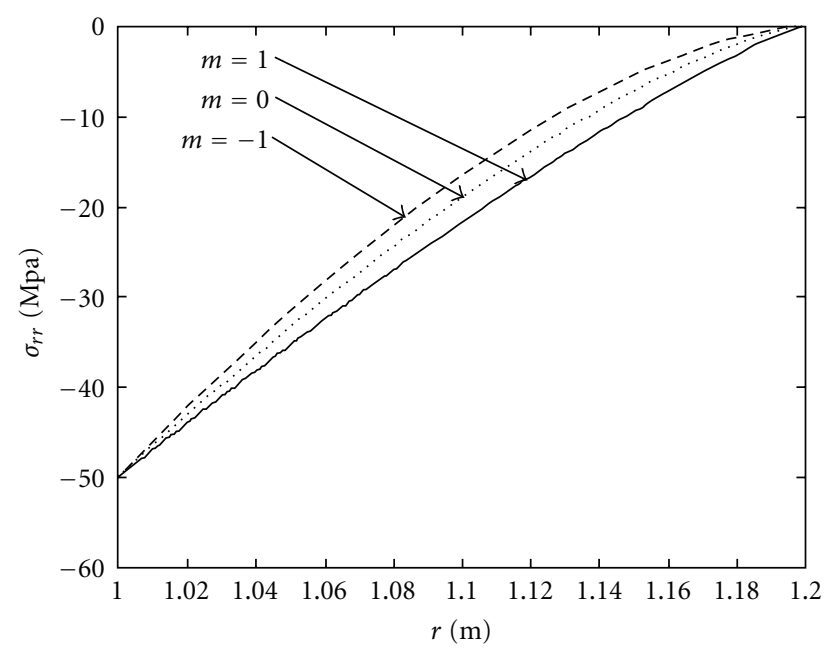

FIGURE 3: Radial stress distribution.

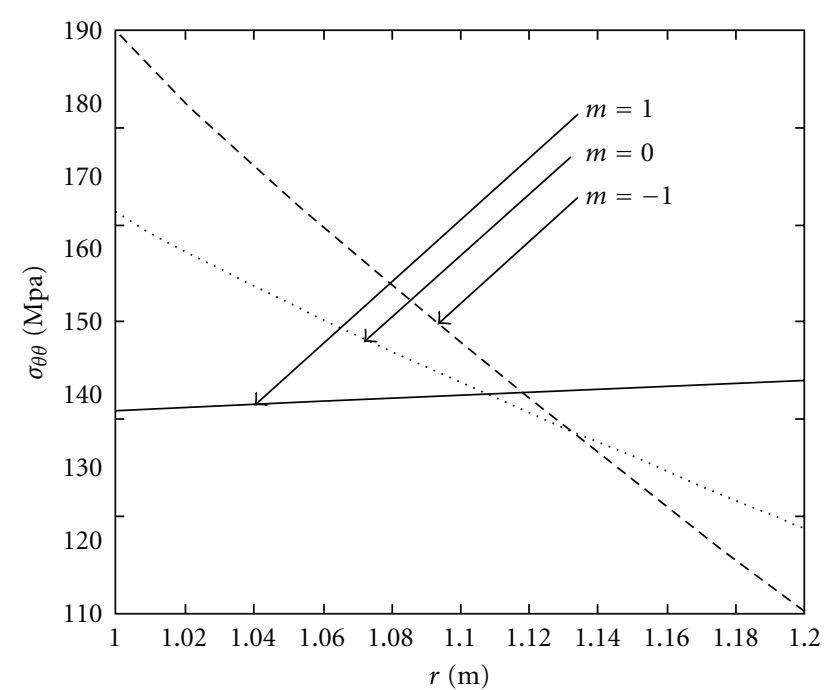

FIgURE 4: Circumferential stress distribution.

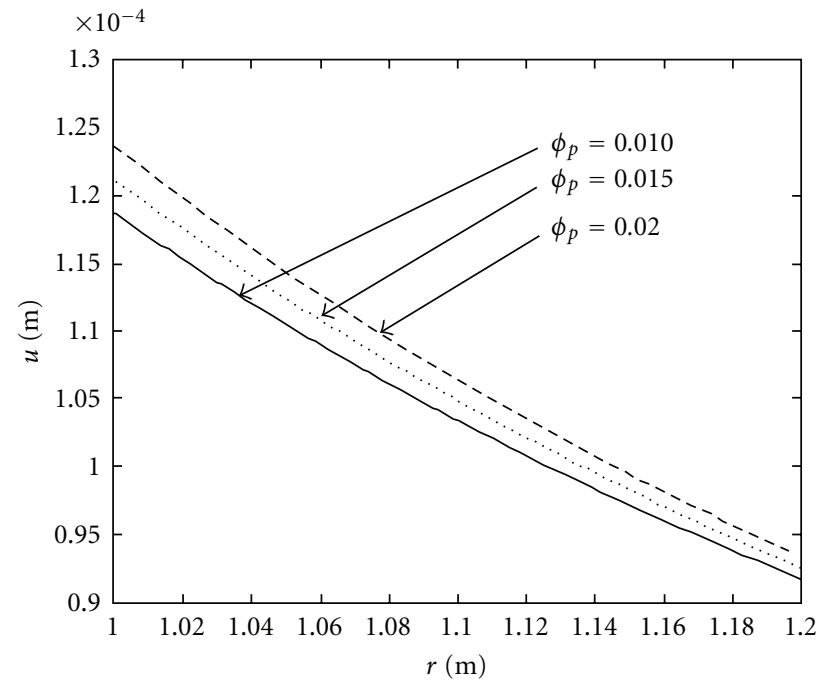

FIGURE 5: Effective porosity on mechanical radial displacement for $m=1$.

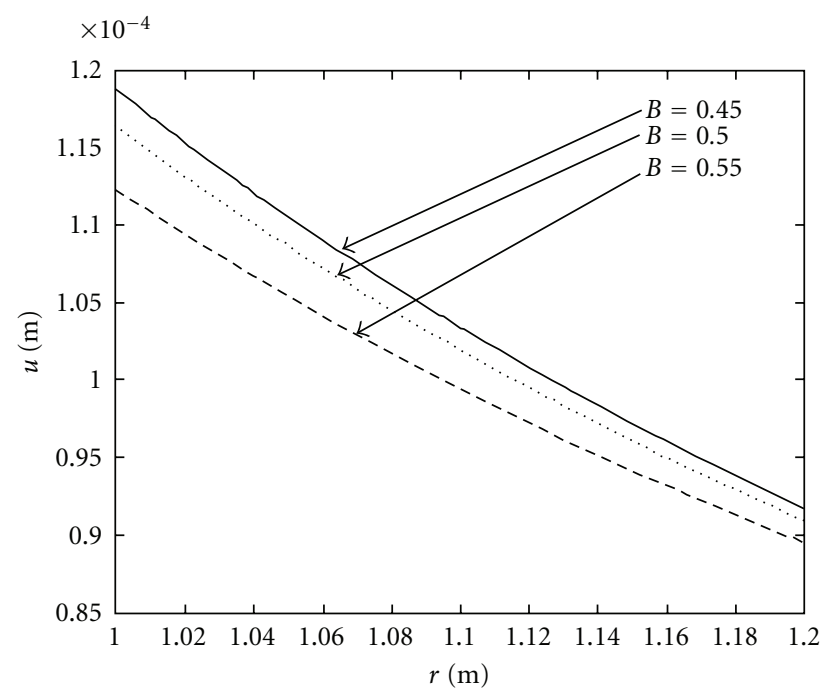

FIGURE 6: Effective compressibility on mechanical radial displacement for $m=1$.

where

$$
\begin{gathered}
c_{1}^{*}=C_{11}+\gamma^{2} m, \\
c_{2}^{*}=2\left(C_{12}+\gamma^{2} m\right), \\
c_{3}^{*}=C_{12}+\gamma^{2} m, \\
c_{4}^{*}=C_{22}+C_{23}+2 \gamma^{2} m .
\end{gathered}
$$

The sphere's material is assumed to be described with a power law function of the radial direction as

$$
\begin{aligned}
c_{i}^{*} & =\bar{c}_{i}^{*} r^{m}, \\
z_{i} & =\bar{z}_{i} r^{2 m},
\end{aligned}
$$

where $m$ is the power law index of the material. 


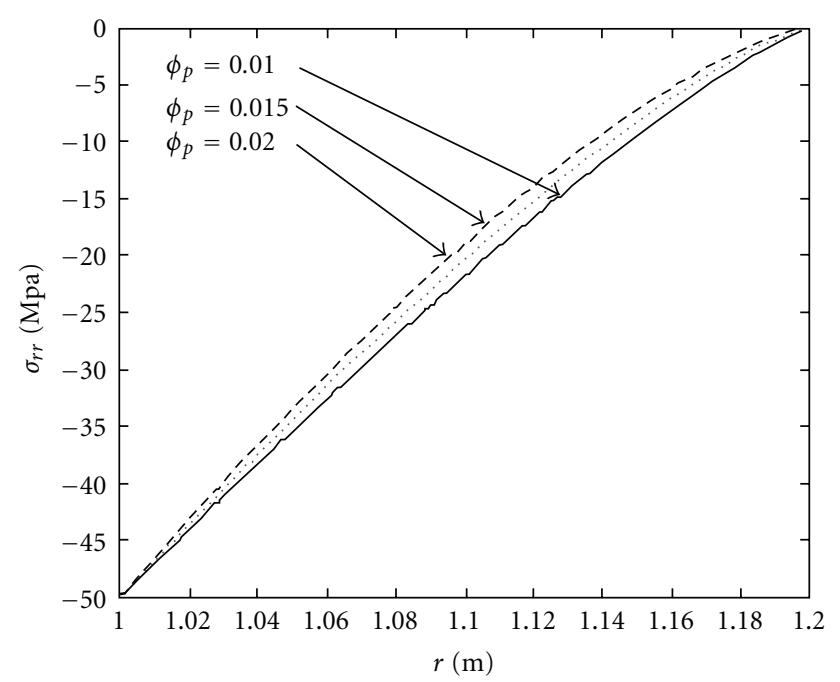

FIGURE 7: Effective porosity on radial stress for $m=1$.

Using relations (1)-(9), the Navier equation in term of the displacement is

$$
\begin{aligned}
& u_{, r r}+\frac{1}{r}\left(\frac{(m+2) \bar{c}_{1}^{*}+\bar{c}_{2}^{*}-2 \bar{c}_{3}^{*}}{\bar{C}_{1}^{*}}\right) u_{, r} \\
& +\frac{1}{r^{2}}\left(\frac{(m+1) \bar{c}_{2}^{*}-2 \bar{c}_{4}^{*}}{\bar{c}_{1}^{*}}\right) \\
& =\bar{z}_{r}\left(\bar{c}_{1}^{*}(m+1+2 L) r^{m-2}\right. \\
& \left.\quad+2 \bar{c}_{2}^{*}(m+1-L)\right)^{r^{2 m-1}},
\end{aligned}
$$

where

$$
L=\frac{\bar{z}_{\theta}}{\bar{z}_{r}}
$$

\section{Heat Conduction Problem}

The heat conduction equation in the steady-state condition for the one-dimensional problem in spherical coordinates and the thermal boundary conditions for a poro FGM hollow sphere are given, respectively, as

$$
\begin{aligned}
\frac{1}{r^{2}}\left(r^{2} k(r) T^{\prime}(r)\right)^{\prime} & =0, \\
c_{11} T^{\prime}(a)+c_{12} T(a) & =f_{1}, \\
c_{21} T^{\prime}(b)+c_{22} T(b) & =f_{2},
\end{aligned}
$$

where $k=k(r)$ is the thermal conduction coefficient and $c_{i j}$ are either thermal conduction coefficient $k$, or convection coefficient $h$, depending on the type of thermal boundary conditions. The terms $f_{1}$ and $f_{2}$ are known constants on the inside and outside radii. It is assumed that the thermal conduction coefficient $k(r)$ is a power function of $r$ as

$$
k=k(r) r^{m}
$$

Using (13), the heat conduction equation becomes

$$
\frac{1}{r^{2}}\left[r^{m+2} T^{\prime}(r)\right]^{\prime}=0
$$

Integrating (12) twice yields

$$
T(r)=c_{1} r^{-(m+1)}+c_{2}
$$

Using the boundary conditions (16) and (17) to determine the constants $c_{1}$ and $c_{2}$ yields

$$
\begin{gathered}
c_{1}=\frac{c_{22} f_{1}-c_{12} f_{2}}{c_{12}\left((m+1) c_{21} b^{-(m+2)}-c_{22} b^{-(m+1)}\right)-c_{22}\left((m+1) c_{11} a^{-(m+1)}-c_{11} a^{-(m+1)}\right)}, \\
c_{2}=\frac{f_{1}\left((m+1) c_{21} b^{-(m+2)} c_{22} b^{-(m+1)}\right)-f_{2}\left((m+1) c_{11} a^{-(m+2)}-c_{12} a^{-(m+1)}\right)}{c_{12}\left((m+1) c_{21} b^{-(m+2)} c_{22} b^{-(m+1)}\right)-c_{22}\left((m+1) c_{11} a^{-(m+2)}-c_{12} a^{-(m+1)}\right)}
\end{gathered}
$$

\section{Solution of the Navier Equation}

The Navier equation for the radial displacement $u$ was given in (10). Equation (10) is the nonhomogeneous Euler differential equation with the general and particular solutions. The general solution, $u_{g}$, is obtained by assuming

$$
u_{g}(r)=B r^{\eta}
$$

Substituting (18) into the homogeneous form of (10) yields

$$
\eta^{2}+\left(\frac{(m+1) \bar{c}_{1}^{*}+\bar{c}_{2}^{*}-2 \bar{c}_{3}^{*}}{\bar{c}_{1}^{*}}\right) \eta+\left(\frac{(m+1) \bar{c}_{2}^{*}-2 \bar{c}_{4}^{*}}{\bar{c}_{1}^{*}}\right)=0,
$$

where (19) has two real roots $\eta_{1}$ and $\eta_{2}$ as

$$
\eta_{1,2}=\frac{-\left((m+1) \bar{c}_{1}^{*}+\bar{c}_{2}^{*}+2 \bar{c}_{3}^{*}\right) \pm \sqrt{\left((m+1) \bar{c}_{1}^{*}+\bar{c}_{2}^{*}+2 \bar{c}_{3}^{*}\right)^{2}-4 \bar{c}_{1}^{*}\left((m+1) \bar{c}_{2}^{*}-2 \bar{c}_{4}^{*}\right)}}{2 \bar{c}_{1}^{*}}
$$


TABLE 1: Matrial constants.

\begin{tabular}{lc}
\hline Elastic constants & Poro constants \\
\hline$C_{11}=1.867 \times 10^{11}$ & $\gamma=0.27$ \\
$C_{12}=0.819 \times 10^{11}$ & $k_{u}=4.1 \times 10^{10}$ \\
$C_{22}=1.628 \times 10^{11}$ & $k=3.5 \times 10^{10}$ \\
$C_{23}=0.270 \times 10^{11}$ & $k_{f}=3.3 \times 10^{3}$ \\
$\alpha_{r}=1.2 \times 10^{-6}$ & $B=0.45$ \\
& $\phi_{p}=0.01$ \\
\hline
\end{tabular}

Thus, the general solution is

$$
u_{g}(r)=B_{1} r^{\eta_{1}}+B_{2} r^{\eta_{2}}
$$

The particular solution $u_{p}(r)$ is assumed to have the form

$$
u_{p}(r)=D_{1} r^{m}+D_{2} r^{2 m+1}
$$

Substituting (22) in (10) yields

$$
\begin{aligned}
& \left(m(m-1)+\left(\frac{\left.(m+2) \bar{c}_{1}^{*}+\bar{c}_{2}^{*}-2 \bar{c}_{3}^{*}\right)}{\bar{c}_{1}^{*}}\right) m\right. \\
& \left.\quad+\left(\frac{(m+1) \bar{c}_{2}^{*}+2 \bar{c}_{4}^{*}}{\bar{c}_{1}^{*}}\right)\right) D_{1} r^{m-2} \\
& \quad+\left((2 m+1)(2 m)+\left(\frac{(m+2) \bar{c}_{1}^{*}+\bar{c}_{2}^{*}+2 \bar{c}_{3}^{*}}{\bar{c}_{1}^{*}}\right)\right. \\
& \left.\quad \times(2 m+1)+\left(\frac{(m+1) \bar{c}_{2}^{*}+2 \bar{c}_{4}^{*}}{\bar{c}_{1}^{*}}\right)\right) D_{2} r^{2 m-1} \\
& =\bar{z}_{r}\left(\bar{c}_{1}^{*}(m+1-2 L) r^{m-2}+2 \bar{c}_{2}^{*}(m+1-L) r^{2 m-1}\right) .
\end{aligned}
$$

Equating the coefficients of the identical powers yields

$$
\begin{gathered}
D_{1}=\frac{\bar{z}_{r}\left(\bar{c}_{1}^{*}(m+1)-2 L\right)}{m(m-1) \bar{c}_{1}^{*}+\left((m+2) \bar{c}_{1}^{*}+\bar{c}_{2}^{*}+2 \bar{c}_{3}^{*}\right) m+(m+1) \bar{c}_{2}^{*}+2 \bar{c}_{4}^{*},} \\
D_{2}=\frac{\bar{z}_{r}\left(2 \bar{c}_{2}^{*}(m+1-L)\right)}{(2 m+1)(2 m) \bar{c}_{1}^{*}+\left((m+2) \bar{c}_{1}^{*}+\bar{c}_{2}^{*}+2 \bar{c}_{3}^{*}\right)(2 m+1)+\left((m+1) \bar{c}_{2}^{*}+2 \bar{c}_{4}^{*}\right)} .
\end{gathered}
$$

The complete solution for $u(r)$ is the sum of the general and particular solutions as

$$
u(r)=u_{g}(r)+u_{p}(r)
$$

Thus,

$$
u(r)=B_{1} r^{\eta_{1}}+B_{2} r^{\eta_{2}}+D_{1} r^{m}+D_{2} r^{2 m+1}
$$

Substituting (27) into (1) and (2), the strains and stresses are obtained as

$$
\begin{aligned}
& \varepsilon_{r r}= B_{1} \eta_{1} r^{\eta_{1}-1}+B_{2} \eta_{2} r^{\eta_{2}-1}+D_{1} m r^{m-1}+D_{2}(2 m+1) r^{2 m}, \\
& \varepsilon_{\theta \theta}=B_{1} r^{\eta_{1}-1}+B_{2} r^{\eta_{2}-1}+D_{1} r^{m-1}+D_{2} r^{2 m} \\
& \sigma_{r r}=\bar{c}_{1}^{*} {\left[B_{1}\left(\eta_{1}+h\right) r^{\eta_{1}-1}+B_{2}\left(\eta_{2}+h\right) r^{\eta_{2}-1}\right.} \\
&+ D_{1}(m+h) r^{m-1}+D_{2}(2 m+1+h) r^{2 m} \\
&\left.-S\left(\bar{c}_{1}^{*} r^{-(m+1)}+\bar{c}_{2}^{*}\right)\right], \\
& \sigma_{\theta \theta}=\bar{c}_{1}^{*} {\left[B_{1}\left(\eta_{1}+f\right) r^{\eta_{1}-1}+B_{2}\left(\eta_{2}+f\right) r^{\eta_{2}-1}\right.} \\
&+D_{1}(m+f) r^{m-1}+D_{2}(2 m+1+f) r^{2 m} \\
&\left.-g\left(\bar{c}_{1}^{*} r^{-(m+1)}+\bar{c}_{2}^{*}\right)\right],
\end{aligned}
$$

where

$$
\begin{gathered}
h=\frac{\bar{c}_{2}^{*}}{\bar{c}_{1}^{*}}, \\
S=\frac{\bar{z}_{r}}{\bar{c}_{1}^{*}}, \\
f=\frac{\bar{c}_{4}^{*}}{\bar{c}_{3}^{*}}, \\
g=\frac{\bar{z}_{\theta}}{\bar{c}_{3}^{*}} .
\end{gathered}
$$

To determine the constants $B_{1}$ and $B_{2}$, the boundary conditions for stresses may be used. Consider the mechanical boundary conditions on the inside and outside radii as

$$
\sigma_{r r}(a)=-P_{a}, \quad \sigma_{r r}(b)=-P_{b} .
$$

\section{Numerical Results and Discussion}

The analytical solution obtained in the previous section may be checked for a one of example. Then, consider a thick hollow sphere of inner radius $a=1 \mathrm{~m}$, and the outer radius $b=1.2 \mathrm{~m}$ of poro FGM material with properties are given in Table 1. The boundary conditions for temperature are taken as $T(a)=10^{\circ} \mathrm{C}$ and $T(b)=0^{\circ} \mathrm{C}$. The hollow sphere may be assumed to be under internal pressure of $50 \mathrm{MPa}$ and zero external pressure $\left(\sigma_{r r}(a)=-50 \mathrm{MPa}\right.$ and $\sigma_{r r}(b)$ $=0 \mathrm{MPa})$. For different values of $m$, temperature profile, 


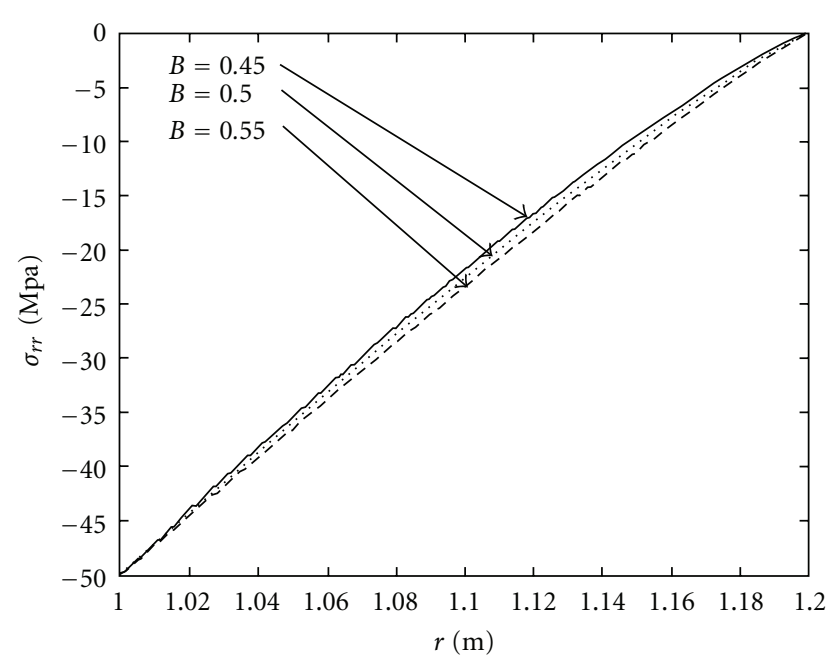

FIGURE 8: Effective compressibility on radial stress for $m=1$.

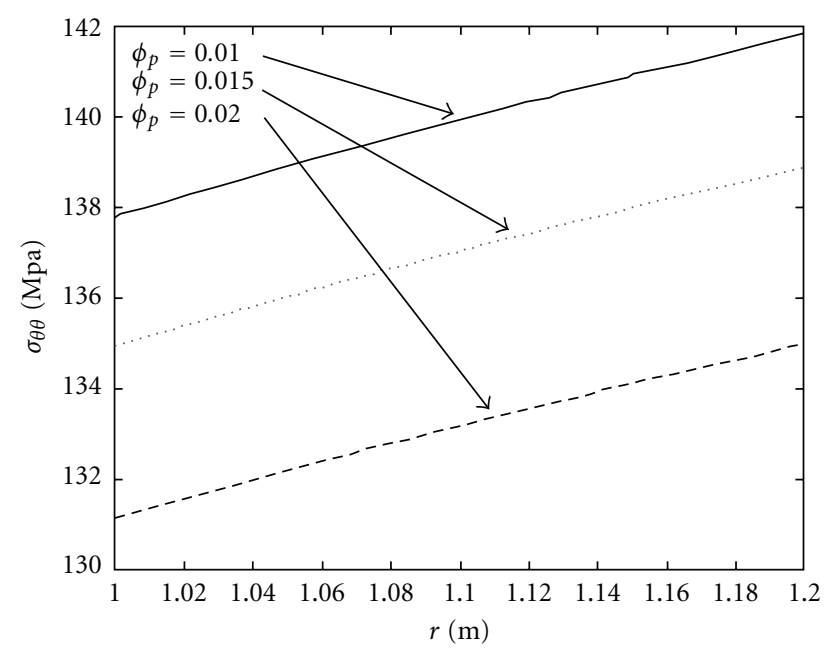

FIGURE 9: Effective porosity on circumferential stress for $m=1$.

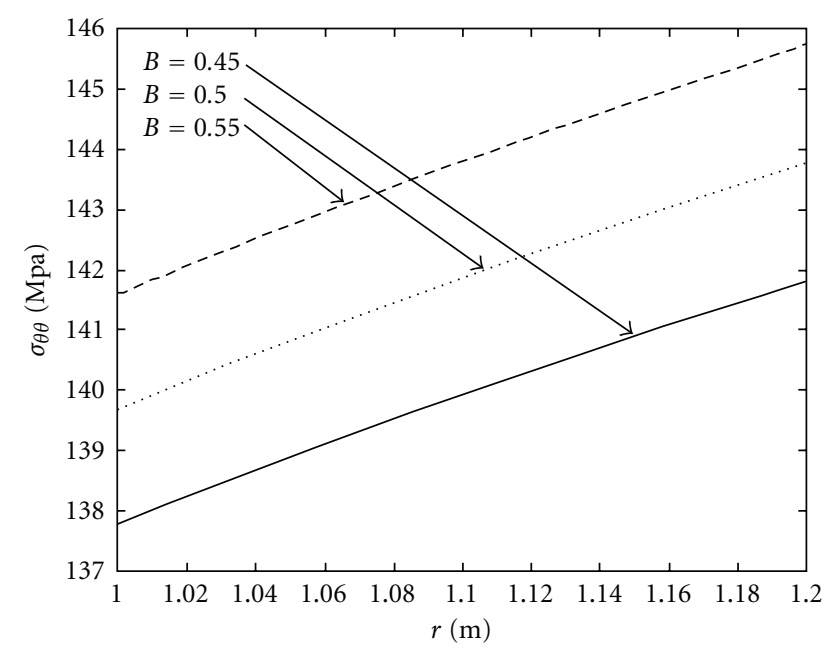

FIGURE 10: Effective compressibility on circumferential stress for $m=1$. radial displacement, radial stresses, and hoop stresses along the radial direction are plotted in Figures 1-4. Figure 1 shows that as the power law increases, the temperature decreases. Figure 2 shows that for higher values of $m$, radial displacement decreases. Figure 3 represents the radial stress along the radial direction and decreases as the power law index increases. The circumferential stress versus the radial direction is shown in Figure 4. It is seen that for $m<1$, the circumferential stress decreases along the radial direction. When $m>1$, the situation is reversed, and the circumferential stress increases along the radial direction. The curve associated with $m=1$ shows that the variation of circumferential stress along the radial direction is minor and is almost uniform across the radius. Effective porosity and compressibility on mechanical radial displacement in Figures 5 and 6, radial stress in Figures 7 and 8 and hoop stress in Figures 9 and 10 for $m=1$ are shown. As noted from Figures 5-10, distribution that with increasing porosity increases radial displacement, and all mechanical stresses decrease. Also, with increasing compressibility, radial displacement decreases, and all mechanical stresses increases.

\section{References}

[1] Q.-H. Qin, Fracture Mechanics of Piezoelectric Materials, WIT, Southampton, UK, 2001.

[2] R. Poultangari, M. Jabbari, and M. R. Eslami, "Functionally graded hollow spheres under non-axisymmetric thermomechanical loads," International Journal of Pressure Vessels and Piping, vol. 85, no. 5, pp. 295-305, 2008.

[3] M. P. Lutz and R. W. Zimmerman, "Thermal stresses and effective thermal expansion coefficient of a functionally gradient sphere," Journal of Thermal Stresses, vol. 19, no. 1, pp. 39-54, 1996.

[4] R. W. Zimmerman and M. P. Lutz, "Thermal stresses and thermal expansion in a uniformly heated functionally graded cylinder," Journal of Thermal Stresses, vol. 22, no. 2, pp. 177$188,1999$.

[5] Y. Obata and N. Noda, "Steady thermal stresses in a hollow circular cylinder and a hollow sphere of a functionally gradient material," Journal of Thermal Stresses, vol. 17, no. 3, pp. 471487, 1994.

[6] Y. Obata and N. Noda, "Transient thermal stress in a plate of a functionally gradient materials," Ceramic Transactions, vol. 34, p. 403, 1993.

[7] Y. Ootao and Y. Tanigawa, "Three-dimensional transient thermal stress analysis of a nonhomogeneous hollow sphere with respect to rotating heat source," Transactions of the Japan Society of Mechanical Engineers A, vol. 60, no. 578, pp. 22732279, 1994 (Japanese).

[8] M. Jabbari, S. Sohrabpour, and M. R. Eslami, "Mechanical and thermal stresses in a functionally graded hollow cylinder due to radially symmetric loads," International Journal of Pressure Vessels and Piping, vol. 79, no. 7, pp. 493-497, 2002.

[9] M. Jabbari, S. Sohrabpour, and M. R. Eslami, "General solution for mechanical and thermal stresses in a functionally graded hollow cylinder due to nonaxisymmetric steady-state loads," Journal of Applied Mechanics, vol. 70, no. 1, pp. 111118, 2003.

[10] Z. S. Shao and T. J. Wang, "Three-dimensional solutions for the stress fields in functionally graded cylindrical panel 
with finite length and subjected to thermal/mechanical loads," International Journal of Solids and Structures, vol. 43, no. 13, pp. 3856-3874, 2006.

[11] J. B. Cheung, T. S. Chen, and K. Thirumalai, "Transient thermal stresses in a sphere by local heating," Journal of Applied Mechanics, vol. 41, no. 4, pp. 930-934, 1974.

[12] Y. Takeuti and Y. Tanigawa, "Transient thermal stresses of a hollow sphere due to rotating heat source," Journal of Thermal Stresses, vol. 5, no. 3-4, pp. 283-298, 1982.

[13] U. Meyer, A. Larsson, H. P. Hentz, and R. A. Caruso, "Templating of porous polymeric beads to form porous silica and titania spheres," Advanced Materials, vol. 14, no. 23, pp. 1768-1769, 2002.

[14] A. Stein, "Sphere templating methods for periodic porous solids," Microporous and Mesoporous Materials, vol. 44, pp. 227-236, 2001.

[15] P. Delage, N. Sultan, and Y. J. Cui, "On the thermal consolidation of Boom clay," Canadian Geotechnical Journal, vol. 37, no. 2, pp. 343-354, 2000.

[16] L. R. Jing and X. T. Feng, "Numerical modeling for coupled thermo-hydro-mechanical and chemical processes (THMC) of geological media-International and Chinese experiences," Chinese Journal of Rock Mechanics and Engineering, vol. 22, no. 10, pp. 1704-1715, 2003.

[17] C. A. Kodres, "Moisture-induced pressures in concrete airfield pavements," Journal of Materials in Civil Engineering, vol. 8, no. 1, pp. 41-50, 1996.

[18] N. Tanaka, J. Graham, and T. Crilly, "Stress-strain behaviour of reconstituted illitic clay at different temperatures," Engineering Geology, vol. 47, no. 4, pp. 339-350, 1997.

[19] E. Romero, A. Gens, and A. Lloret, "Suction effects on a compacted clay under non-isothermal conditions," Geotechnique, vol. 53, no. 1, pp. 65-81, 2003.

[20] Y. Wang and E. Papamichos, "An analytical solution for conductive heat flow and the thermally induced fluid flow around a wellbore in a poroelastic medium," Water Resources Research, vol. 36, no. 5, pp. 3375-3384, 1994.

[21] Y. Wang and E. Papamichos, "Thermal effects on fluid flow and hydraulic fracturing from wellbores and cavities in lowpermeability formations," International Journal for Numerical and Analytical Methods in Geomechanics, vol. 23, no. 15, pp. 1819-1834, 1999.

[22] M. Kurashige, "A thermoelastic theory of fluid-filled porous materials," International Journal of Solids and Structures, vol. 25, no. 9, pp. 1039-1052, 1989. 

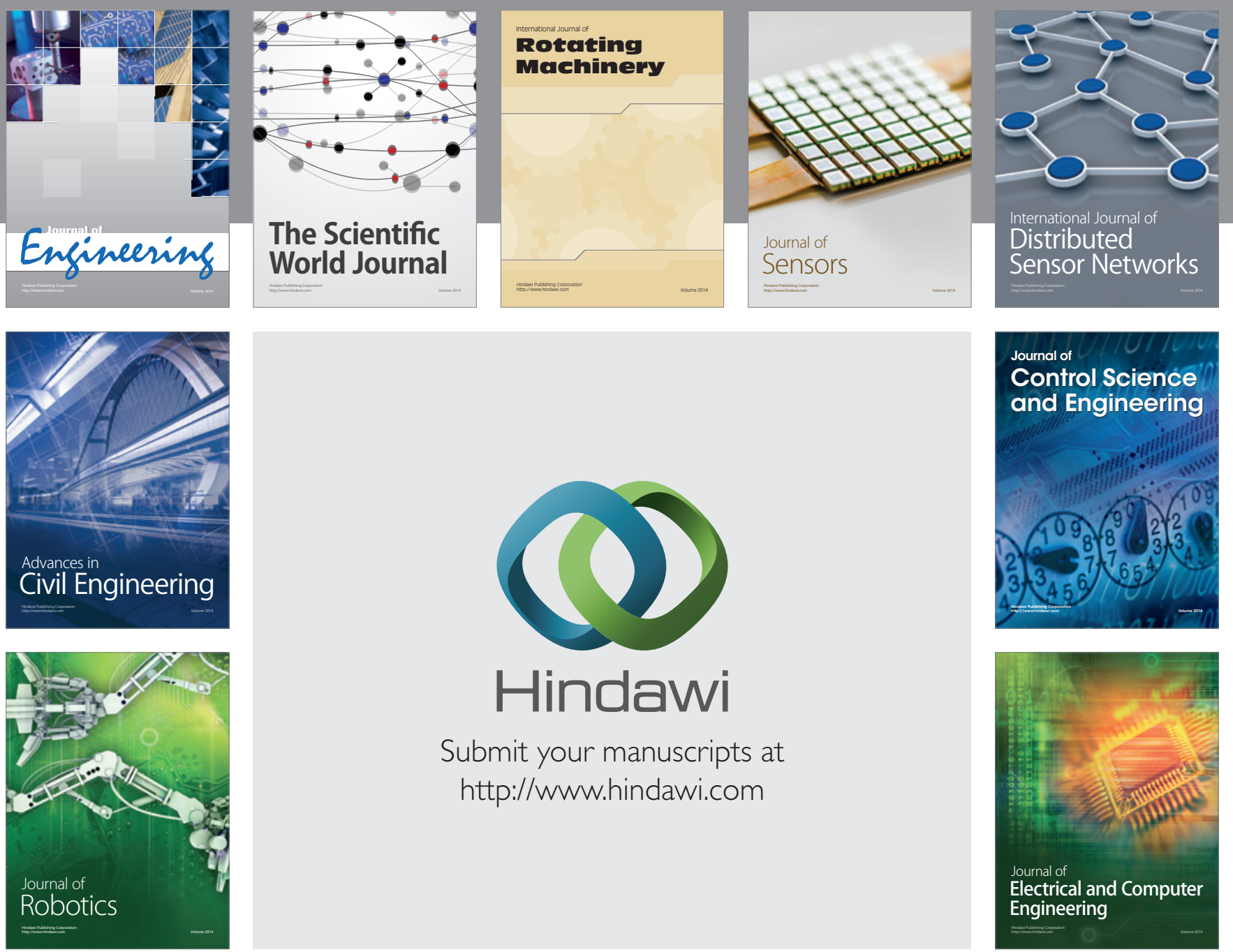

Submit your manuscripts at

http://www.hindawi.com
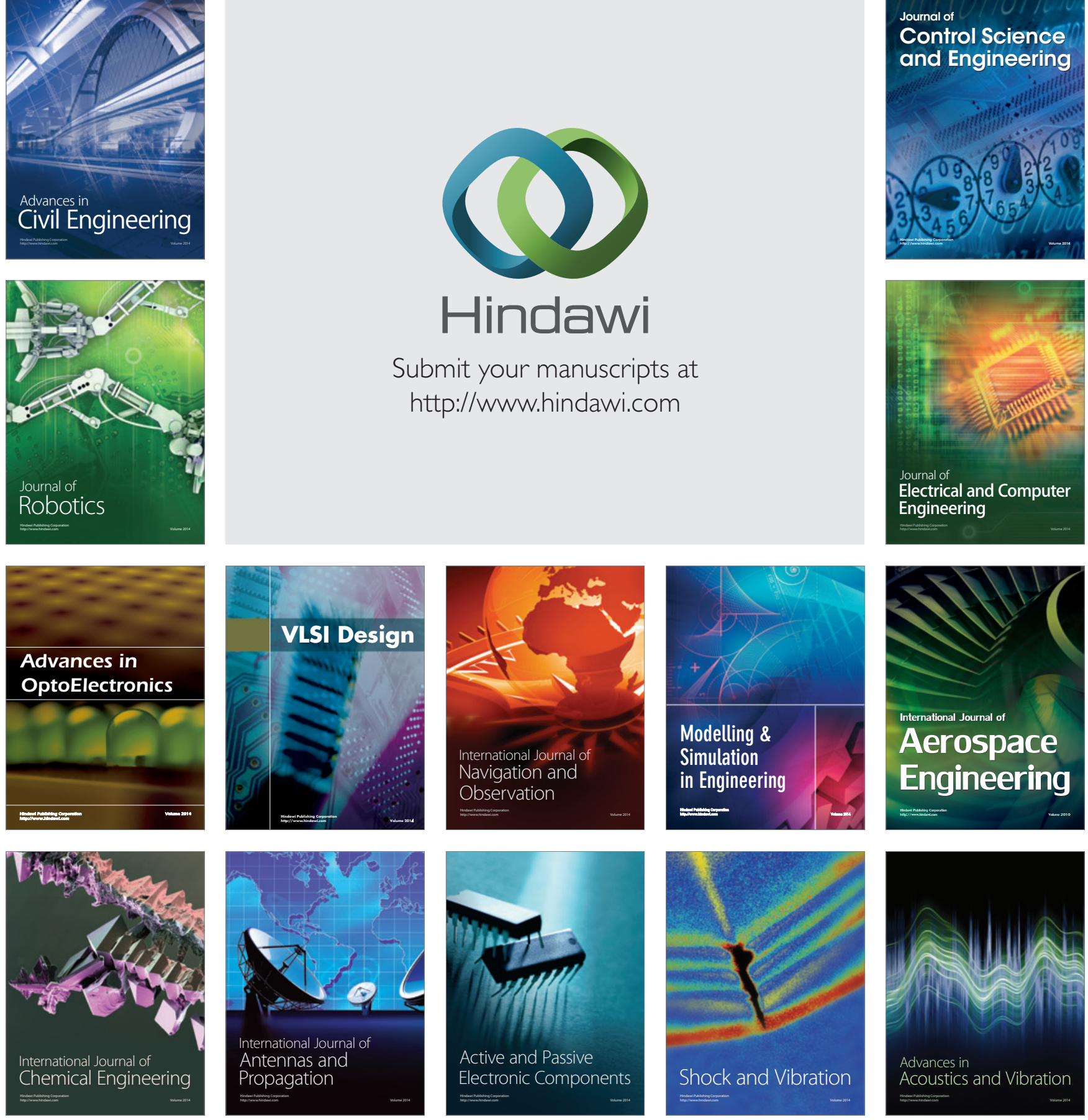